\title{
estado actual de la automación [*]
}

\author{
P. WEBER, DE NEUBECKUM (**) \\ Dr. Ingeniero
}

\section{1. introducción}

Hace unos diez años que se consiguió realizar con éxito la primera regulación automática de un horno Lepol con enfriador Fuller 1). Basándose en tales principios, se ha logrado hoy la regulación, no sólo de los hornos Lepol, sino también de los hornos con precalentamiento en suspensión gaseosa. Tal procedimiento es susceptible, asimismo, de utilización en el control y cálculo del proceso en los hornos.

\section{2. fundamentos y características acerca de la regulación de hornos}

Para una mejor comprensión, volveremos a recordar los criterios básicos para la regulación de hornos (fig. 1).

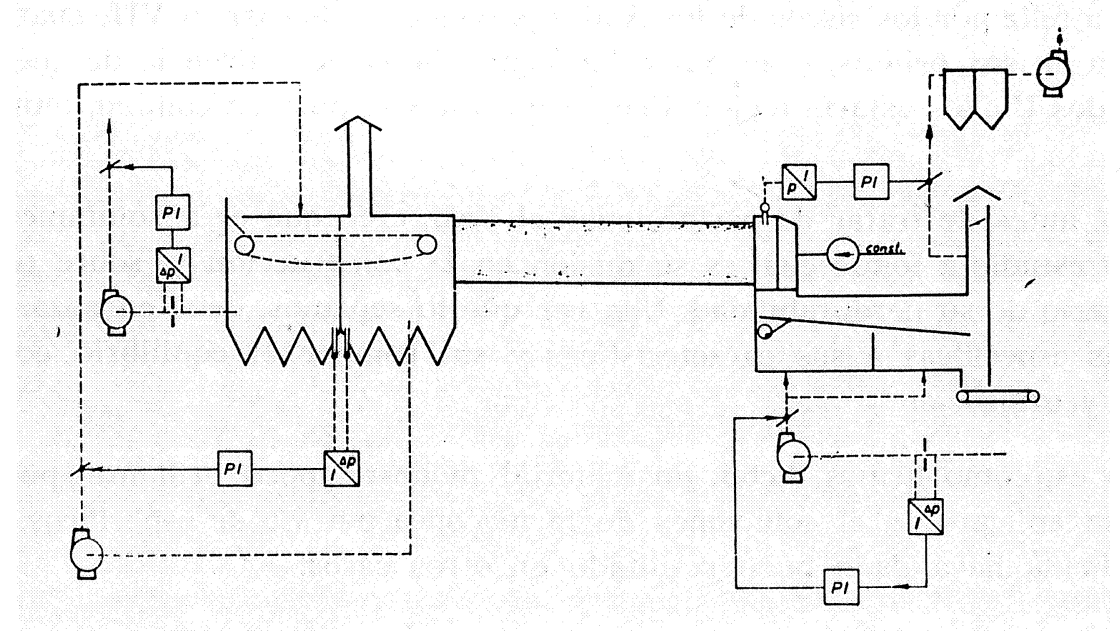

Fig. 1.-Regulación de un horno Lepol.

* Informe ante la reunión plenaria de la Asamblea "Calor y Energía» de la Asociación de Fabricantes de Cemento, de Alemania, celebrada el 16 de febrero de 1966, en Düsseldorf.

** Este artículo es una transcripción del publicado en Zement-Kalk-Gips año LVI (enero 1967), cuaderno 1, página 6 , por el Dr. Weber, a quien agradecemos la posibilidad de incluir su trabajo en esta publicación. 
La composición y volumen del crudo en polvo condicionan la cantidad de calor que ha de aplicarse en cada punto del horno. De lo que se deduce que, con una composición constante y un flujo permanente del crudo, las relaciones entre la corriente de material y las temperaturas de aire y gas se mantendrán constantes en todos los puntos del horno, ya que regulan la transmisión térmica.

Esta situación óptima para la explotación de cada horno deberá determinarse mediante mediciones y experiencias. Las temperaturas y volúmenes de corriente que se obtengan de las mismas, deberán mantenerse adoptando las medidas de regulación correspondientes.

En el horno Lepol, esto puede conseguirse manteniendo constante:

1) la alimentación de polvo crudo;

2) el volumen de gases de escape;

3) el volumen del aire en el enfriador;

4) la corriente gaseosa entre las cámaras I y II de la parrilla mediante la regulación por diferencia de presión;

5) el volumen de combustible.

El criterio básico de este procedimiento de regulación corresponde al «modelo estático» de un programa de cálculo para hornos rotatorios, es decir, la regulación para una situación ideal de explotación.

La condición previa más importante es la composición constante del crudo, puesto que ella condiciona las exigencias térmicas y las temperaturas necesarias para el horno rotatorio. Pero si, por el contrario, existen oscilaciones en la composición del polvo crudo, deberán establecerse, con la mayor rapidez posible, sus repercusiones sobre el proceso del horno, valiéndose de un computador rápido, controlándolas mediante la oportuna modificación de los factores nominales. El computador trabaja entonces según un «modelo dinámico».

Las experiencias realizadas en este sentido en U.S. A. no han transcurrido hasta hoy de forma satisfactoria, y se presume que no será posible disponer, dentro de un plazo previsible, de un dispositivo de cálculo capaz de dominar las numerosas dificultades que se presentan.

En principio, es evidentemente mucho más sencillo: mantener la calidad del crudo en polvo. Con esto se obtienen, de paso, las siguientes ventajas:

1) regulación más sencilla del horno;

2) proceso de horno más regular;

3) calidad de clínker más uniforme;

4) control más sencillo de la molienda del cemento;

5) limitación de los costes de inversión para una automación total. 


\section{3. composición uniforme del polvo crudo}

Un crudo uniforme, no sólo debe ser constante en su contenido de $\mathrm{CaCO}_{3}$, sino también: en sus módulos, principalmente en lo que se refiere al standard de cal. En el caso de que entren a formar parte de él diversos ingredientes capaces de sufrir variaciones en su composición, el procedimiento de análisis rápido se hace todavía más necesario.

En la práctica sólo se necesita un aparato de análisis por fluorescencia de rayos $\mathrm{X}$ (denominado abreviadamente aparato RFA ) ${ }^{2}$ y ${ }^{3}$ ). Para las consideraciones que hagamos más. adelante, es importante tener en cuenta que la realización de los ensayos con los aparatos. RFA puede verificarse de modo discontinuo y continuo.

La elección entre ambos procedimientos de medida depende, fundamentalmente:

1) de las propiedades del proceso de medición propiamente dicho (por ejemplo, exactitud y gastos de entretenimiento y servicio);

2) de las propiedades y número de las máquinas que han de regularse (incluyendo, por ejemplo, los molinos y maquinaria de cantera).

Una composición uniforme del crudo se alcanzaría, por consiguiente:

1) homogeneizando el material a granel resultante de la operación de cantera, en un lecho mezclador (fig. 2), por medio de análisis desarrollados de forma permanente;

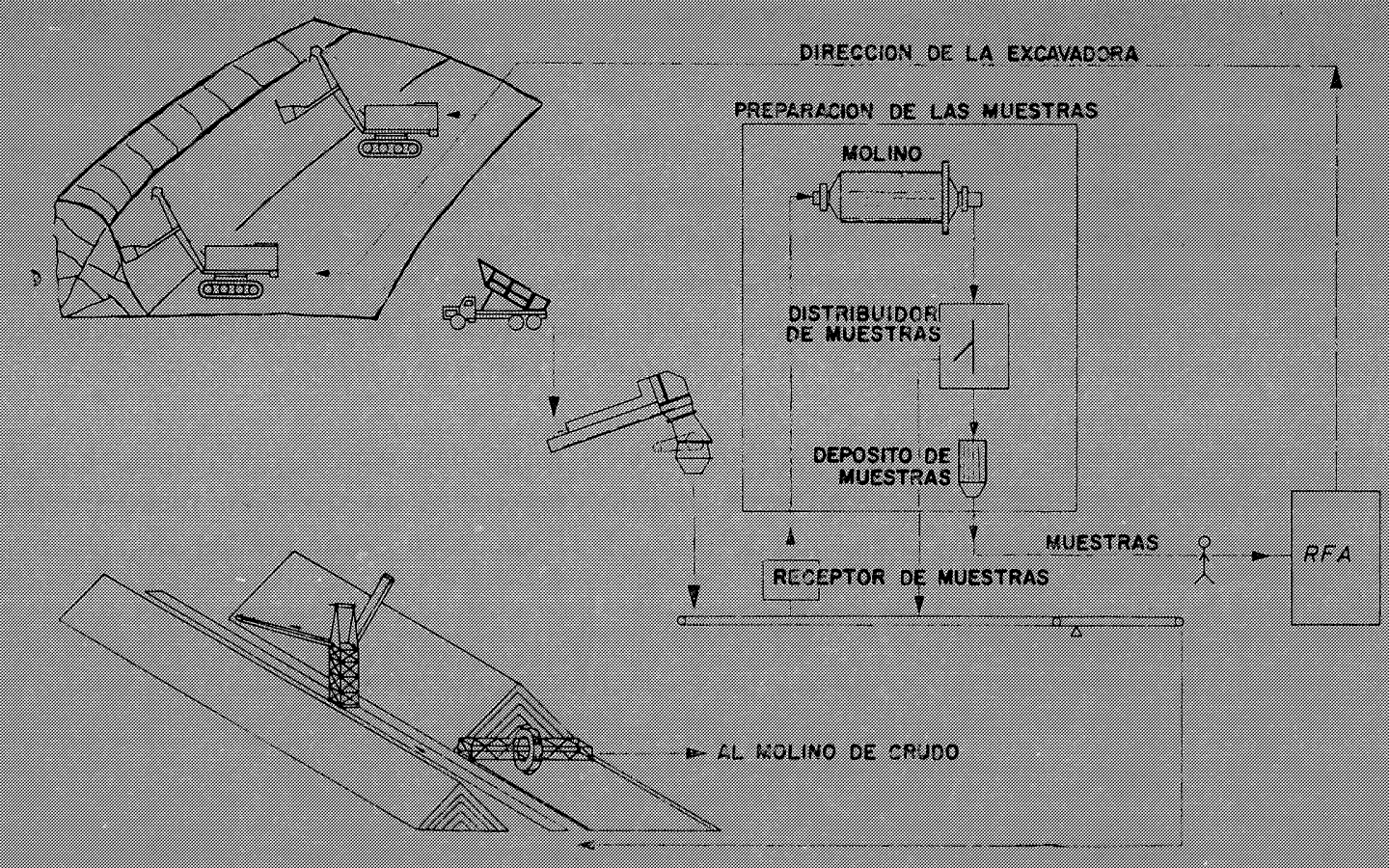

Tig. 2.-Procedimiento del. lecho mezdador, loma do muestras der mismo.

2) controlando los componentes de las materias primas, antes del proceso de molienda, mediante análisis de la mezcla de material a granel, la cual podrá mejorarse todavía más con una homogeneización adicional consecuente a la molienda (fig. 3); 


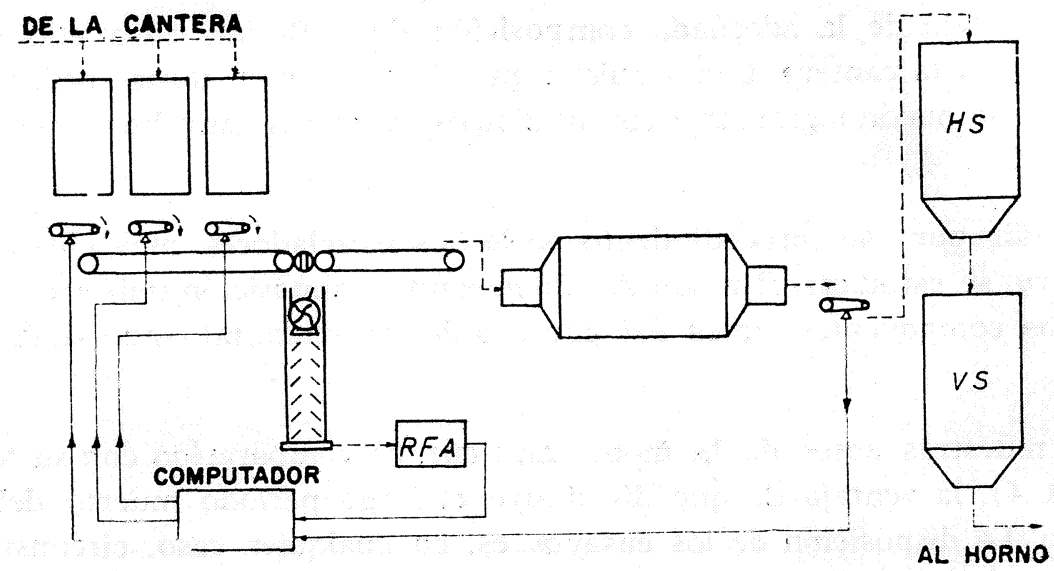

Fig. 3.-Control de componentes, toma de muestras antes del molino.

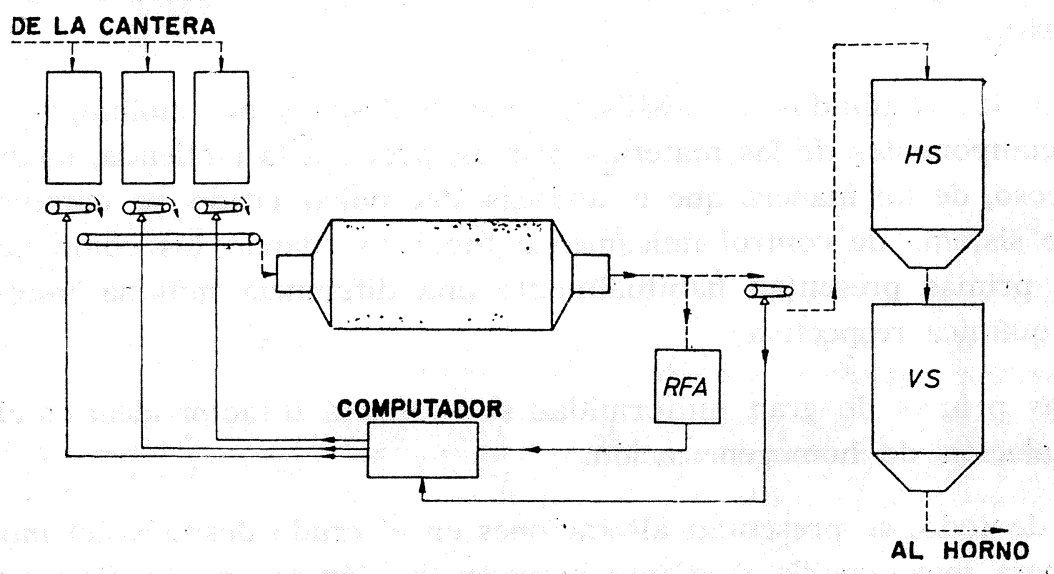

Fig. 4.-Control de componentes, toma de muestras detrás del molino.

3) controlando los componentes del material a granel, antes de la molienda, mediante análisis del polvo crudo, a la salida de molino (fig. 4). Este polvo crudo debe homogeneizarse además posteriormente.

Los lechos mezcladores ${ }^{4}$ ) se emplearán con materias primas muy irregulares y con oscilaciones imprevisibles.

Una de las plantas de fabricación de cemento que ha utilizado este método es la fábrica «Riverside», de California. En Europa se practicó el proceso de lecho mezclador en la fábrica de cementos Val d'Azergues, de Lafarge ${ }^{4}$ ) y en la planta de cemento de Villaluenga, en España. La materia prima se dispone en finas capas horizontales superpuestas en pilas. Sobre el montón así preparado, se trabaja en estratos verticales - la mayoría de las veces por medio de una excavadora de ruedas de paletas- para que pueda obtenerse una buena mezcla. Los montones se disponen para el acopio de materias primas durante una semana aproximadamente.

Del material a granel procedente de cantera se fueron obteniendo pruebas periódicamente, las cuales, una vez desecadas y molidas, se colocaban en un dispositivo de ensayo para análisis. De acuerdo con estos análisis, se verificará la extracción en cantera, de modo 
que el montón disponga de la adecuada composición de material; para ello es necesario hacer perforaciones en la cantera. Como quiera que el acopio de materias primas en el montón es muy grande, se puede lograr, aun con una materia prima muy heterogénea, el factor nominal deseado.

Antes de decidir por este procedimiento de lechos mezcladores, muy efectivo, pero desgraciadamente caro, se estudiará el modo de obtener una composición uniforme de polvo crudo, manejando los componentes, antes del proceso de molienda, tal como se describe en las. figuras 3 y 4 .

La toma de muestras antes de la molienda, tiene, en comparación con su toma después de la misma (fig. 4), la ventaja de que disminuye el largo período muerto del molino, de media a una hora. La disposición de los ensayos es, en cualquier caso, circunstancial, y requiere, con materias primas secas y de difícil obtención, la mayor parte de las veces, un tiempo tan excesivo, que no permite aprovechar la ventaja conseguida con el acortamiento del tiempo muerto.

Basándose en los resultados del análisis, antes o después del molino, se controlará la relación de los componentes de las materias primas, previa a la molienda, mediante un computador de proceso, de tal manera que el análisis del polvo crudo se corresponda con el valor ideal. Este sistema de control únicamente funciona cuando los componentes aislados de las materias primas presentan habitualmente una diferencia mínima muy uniforme en la composición química respectiva.

Con materias primas de gran uniformidad se alcanzará el factor ideal en el polvo crudo, incluso sin instalación de homogeneización.

Si, a pesar de todo, se presentan alteraciones en el crudo después del molino, se rectifican de la manera más sencilla mediante homogeneización en un silo. Para ello se controla la relación de componentes de las materias primas, en función de lả composición del polvo, en un silo de homogeneización. La composición del crudo correspondiente, en el silo de homogeneización, se indica al computador en función de los volúmenes obtenidos y de su composición química, determinando entonces la disposición necesaria de los componentes.

Existen dos sistemas para el control del crudo en polvo:

1) el aparato discontinuo RFA establece y dispone periódicamente la relación de componentes, valiéndose del proceso de pulsación. La vigilancia continua de la explotación viene asegurada con una toma independiente de muestras, practicada en este espacio de tiempo;

2) el aparato continuo RFA valora las mueștras de un modo continuo, si bien deberá regularse la proporción de componentes, de modo continuo, por el procedimiento de pulsación, condicionado por el tiempo muerto existente entre la regulación y la toma de factores de medición, por su trascendencia.

En el caso de alteraciones en la composición química de los componentes aislados, deberá practicarse una regulación, si bien no hay necesidad de que ésta sea continua, bastando con considerar la composición química media de un material determinada en un sector de tiempo dado, como punto inicial, con el fin de evitar una regulación excesiva. Cuando se trata de componentes uniformes, no hay, por lo tanto, necesidad de recurrir a una regulación demasiado frecuente. 
En consecuencia, los tipos del proceso de regulación son análogos con los aparatos RFA continuos y discontinuos, ya que en ambos casos se prefiere el proceso discontinuo de regulación. La diferencia consiste únicamente en la expresión de los factores que se utilizan como impulsos reguladores. Con el aparato discontinuo RFA se analizará, en el período de tiempo comprendido entre dos maniobras de regulación, una muestra conjunta compuesta de varias aisladas. Con el aparato RFA continuo, el computador selecciona, partiendo de los análisis continuos de que disponga, un número de ellos que servirán para la determinación del factor medio. El número de análisis corresponde, aproximadamente, al de muestras aisladas que integren la muestra conjunta.

El factor medio de ambos procesos es teóricamente análogo. En este sentido ha de tenerse en cuenta la posibilidad de oscilaciones en la aportación de crudo. Como quiera que asimismo se hace necesario, para la regulación, un procedimiento de pulsación, resulta evidente e indicada la aplicación discontinua cuando se prevea el uso de sólo un aparato RFA. Este también es aprovechable para otros cometidos, lo cual a menudo resulta muy favorable para otras circunstancias de explotación.

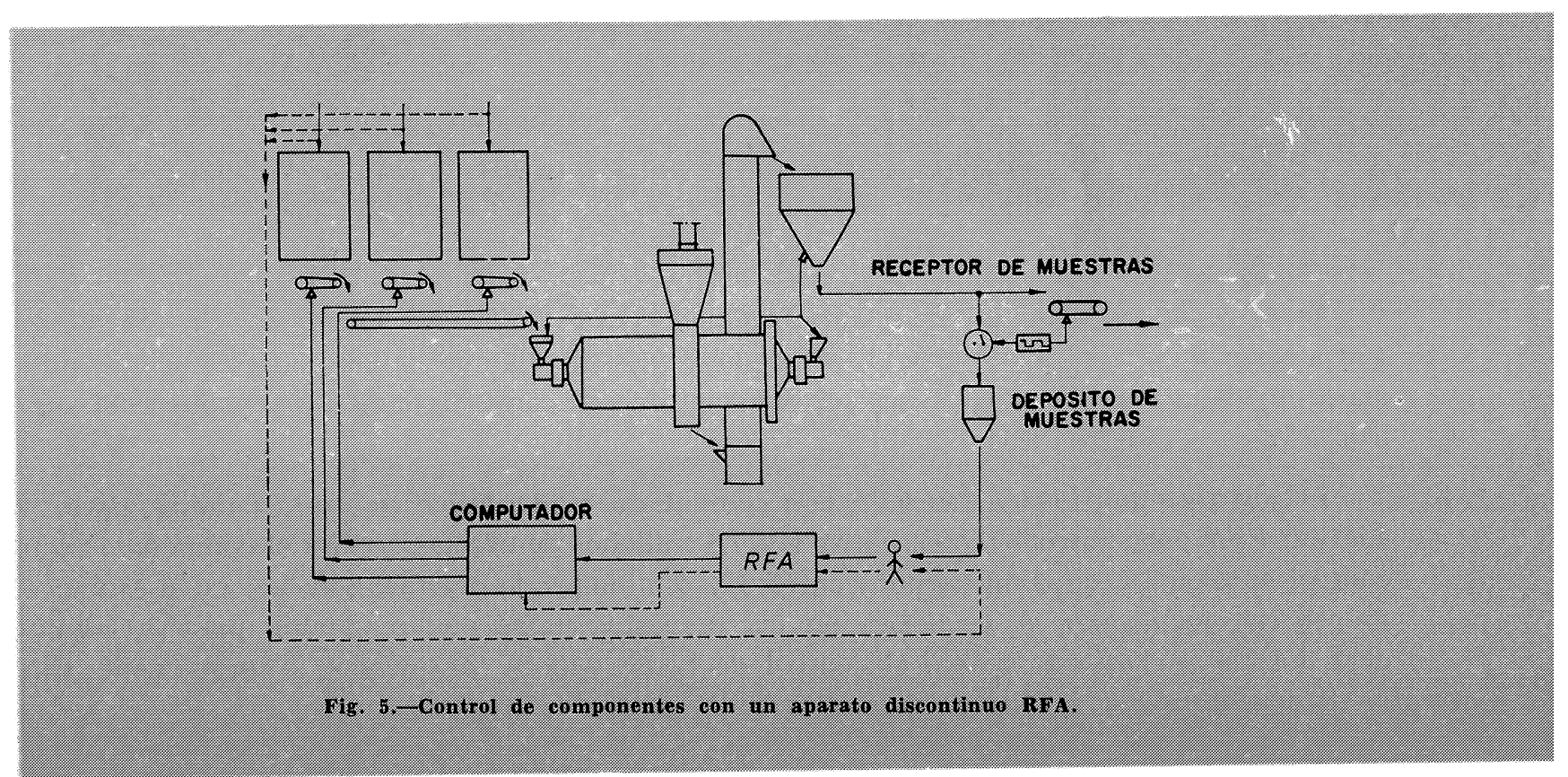

En la figura 5 se muestra el esquema de un control de materias primas con aparato discontinuo RFA. Entre los análisis de crudo se investigarán, también por separado, los componentes de las materias primas, suministrando los resultados al computador, al objeto de incrementar el coeficiente de exactitud del control con dicho computador, disminuyendo las oscilaciones en el crudo resultante.

Si se aplica un aparato continuo RFA para el control de molido, se hace necesario, por lo general, la adopción de un segundo, de tipo discontinuo.

Este procedimiento de regulación se ha seguido, entre otros, en la Compañía de Cemento Portland de California, en su factoría de Colton (fig. 6). El análisis del crudo procedente del molino se verifica con un aparato continuo. Como quiera que éste resulta de más conveniente aplicación, exclusivamente en un punto, se aplicará un segundo aparato discontinuo RFA para el análisis de los componentes de las materias primas. 
La ventaja de aplicar el aparato continuo RFA reside en su simplicidad de aplicación para realizar los ensayos. Es susceptible de utilización con materias primas química y mineralógicamente uniformes, con las cuales, y en condiciones raramente favorables de explotación, puede ser útil en colaboración con otro aparato RFA situado en un punto.

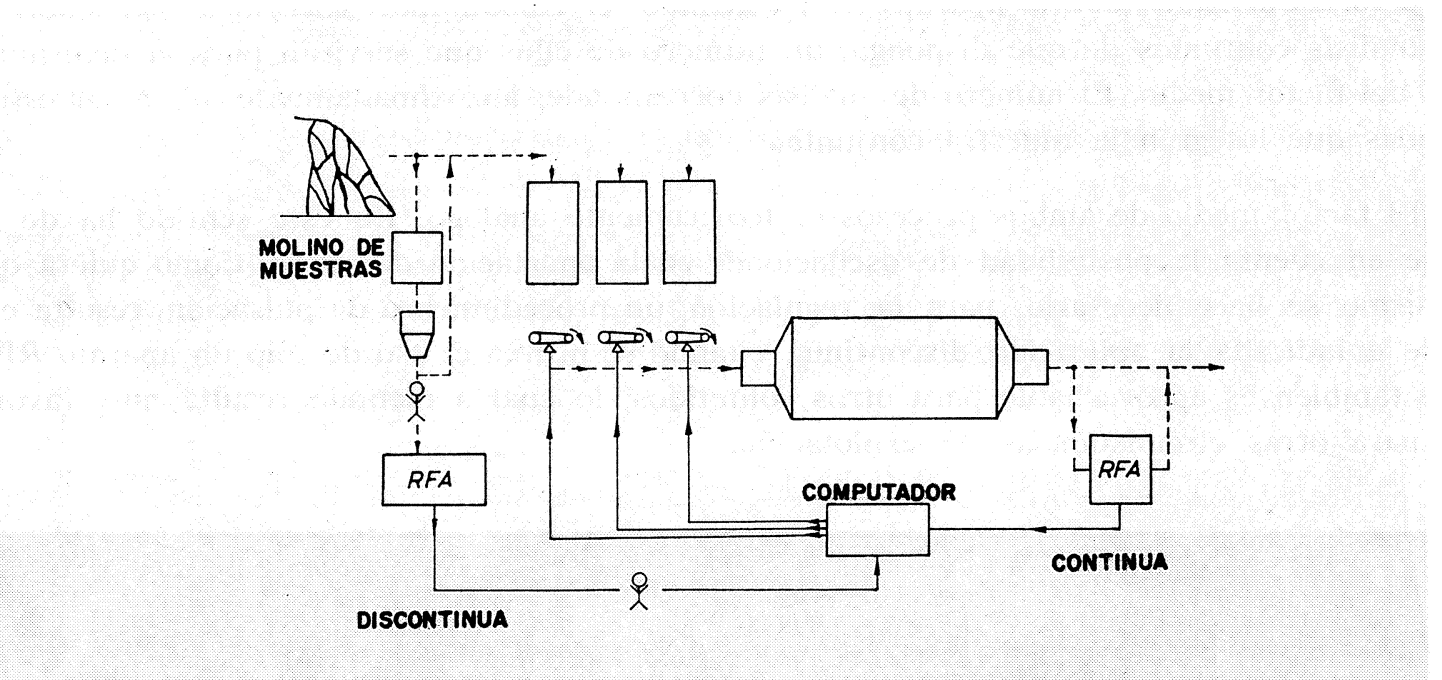

Fig. 6.--Esquema del control de materias primas en la fábrica Colton, de Galifornia.

Antes de la adopción de un aparato RFA, deberán verificarse todas las circunstancias que afecten a la técnica del mismo, al personal y, especialmente, a las materias primas propiamente dichas. Entre otras cosas, el material deberá convertirse en clínker antes del análisis RF.

El costo de una instalación RFA con control de molienda es elevado, y oscila, para el aparato RFA propiamente dicho, entre 3 y 7,5 millones de pesetas, y para el computador con control automático de componentes, entre 3 y 6 millones de pesetas. Esta inversión está, por lo general, justificada, si se tienen en cuenta las ventajas que resultarán para la regulación del horno. Por esta razón, el control de horno deberá considerarse conjuntamente con el sistema de regulación de componentes en el molino de crudo.

\section{4. flujo uniforme del material}

Para la regulación de horno, es condición previa importante, junto a una «composición uniforme del crudo", un flujo constante de material, ya que para cualquier sistema de horno es necesario una alimentación constante de polvo crudo, mediante una báscula de dosificación por cinta.

En el horno Lepol la parrilla constituye un distribuidor volumétrico. Como quiera que el peso por litro, de los gránulos húmedos, disminuye, la distribución volumétrica no suele coincidir con la gravimétrica exigida, lo cual puede dar lugar a que, con una velocidad constante de emparrillado, pueda variar la alimentación.

Para mantener el flujo de material a peso constante puede regularse, como se ha demostrado, la velocidad de la parrilla en función del peso por litro del granulado. Esto se 
verifica de forma que la altura de alimentación del granulado en la tolva se mantenga constante por la regulación de la velocidad de la parrilla.

La figura 7 representa el Gráfico de un control de parrilla Lepol en función de la altura del granulado, el cual permilte reconocer que las oscilaciones en el peso por litro pueden rectificarse con la velocidad de la parrilla.

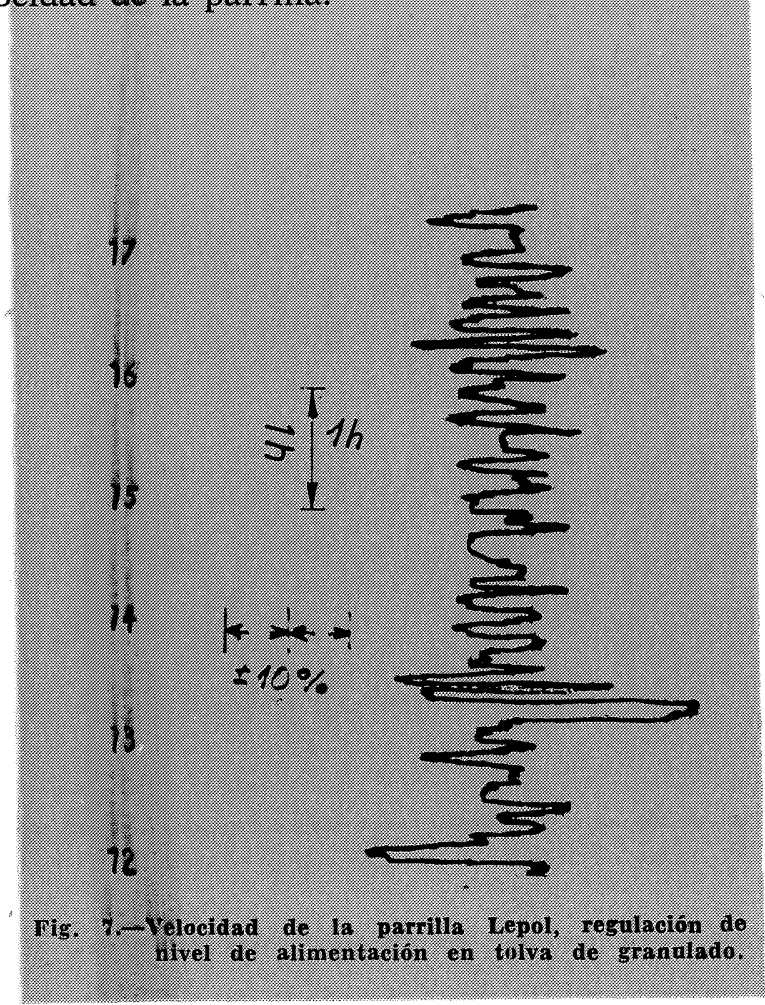

\section{5. volúmenes constantes de gas y aire}

La regulación de las cantidades de gas y aire no representa dificultad alguna para una planificación adecuada de la instalación. En el horno Lepol, los volúmenes de los gases de escape, a consecuencia de las grandes diferencias de resistencia del lecho de parrilla al paso de los gases, oscilan más que en los hornos con precalentamiento en suspensión, cuya resistencia a la corriente está determinada casi exclusivamente por el sistema de precalentamiento y apenas sufre alteraciones. El volumen de gases de escape en los hornos con precalentadores en suspensión se mantiene, por esta razón, bastante constante, incluso sin regulación, siempre que no se modifiquen las resistencias en la zona de compresión del ventilador-exhaustor al aprovechar el calor residual en otra instalación de la fábrica.

Puede conseguirse un comportamiento constante del enfriador mediante una regulación directa del volumen de aire de refrigeración, o manteniendo constantes las diversas presiones y diferencias de presión en el enfriador ${ }^{1}$ ).

A título de ejemplo, véase en la figura 8 el esquema de un enfriador de tres escalones instalado en el Japón y adaptado a un horno Dopol de 2.600 t/día. 
La presión de la cámara I se mantendrá constante, fijando el número de revoluciones del ventilador de alta presión y modificando la velocidad del primer escalón de la parrilla. Como quiera que el régimen de revoluciones del ventilador no se modificará si existe una presión uniforme, el volumen de aire de este ventilador seguirá siendo constante. En un segundo

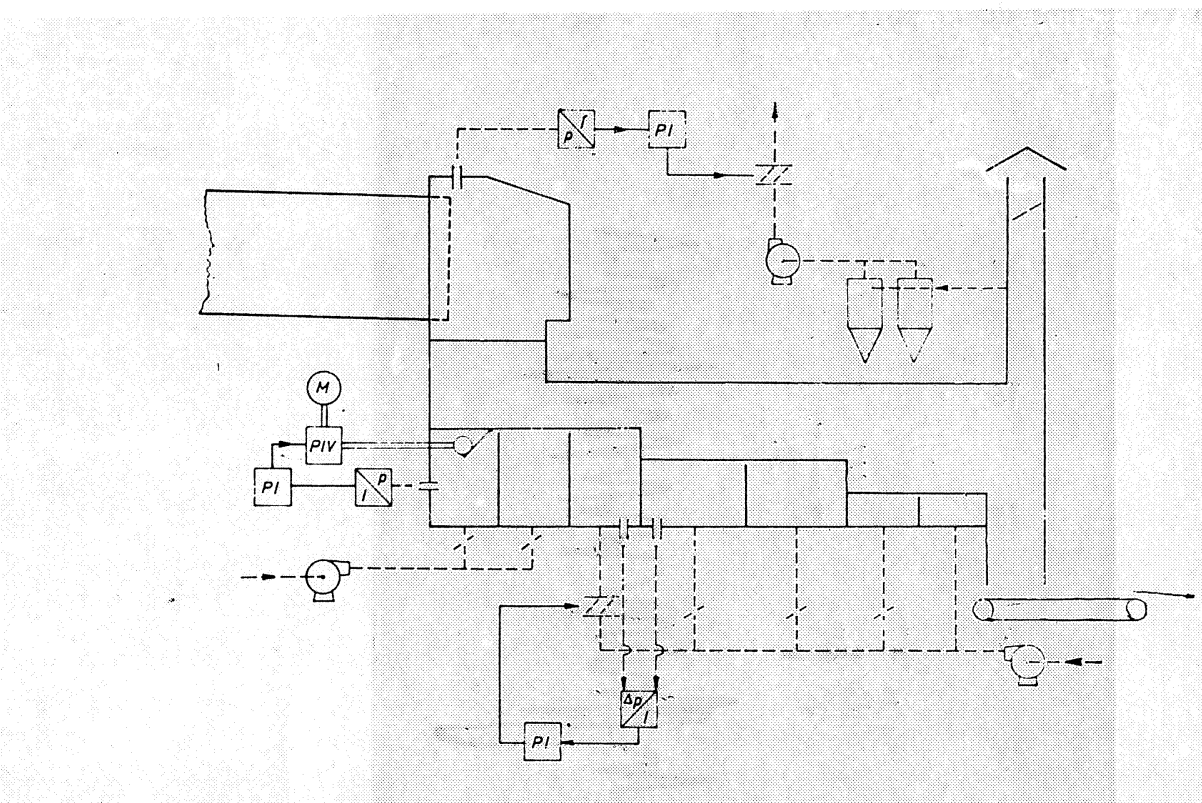

Fig. 8.-Regulación de un enfriador Fuller en un horno japonés Dopol do 2.600 t.

circuito de regulación podrán mantenerse también constantes las presiones diferenciales entre las cámaras III y IV ( $\mathrm{y}$, por tanto, entre el primero y segundo escalones de la parrilla), ya que la corriente de aire entre las cámaras aisladas no se modifica por debajo de estos dos escalones. Por medio del tercer circuito puede regularse la presión en la cabeza de horno, manejando el volumen de aire de refrigeración que escapa a la atmósfera. Si se mantienen constantes las distintas presiones, se obtendrá así una distribución uniforme del aire de enfriamiento sobre cada una de las cámaras, conforme a la primitiva propuesta de Gringmuths ${ }^{1}$ ).

Con estos medios pueden regularse grandes hornos Dopol de $2.600 \mathrm{t} /$ día de rendimiento, con pleno éxito. No hay necesidad de vigilar en ellos el crudo mediante una instalación RFA; sin embargo, debe de homogeneizarse de forma satisfactoria. Las relaciones de escape se mantendrán siempre constantes, llevando a cabo la regulación de enfriamiento, como se ha descrito arriba. Aquí permanecen uniformes las relaciones de corriente y las temperaturas de aire, gas y material, así como el flujo del mismo, con lo cual es posible conseguir en todo momento una capacidad máxima del horno con óptimo aprovechamiento térmico y obtención de un clínker de calidad uniformemente buena.

Resulta evidente, que es factible conseguir una regulación automática del horno mediante la elección de sistemas de mayor simplicidad relativa, principalmente cuando se pretenda evitar la mayor parte de las influencias nocivas para el crudo, sin necesidad de utilizar imprescindiblemente un conmutador de proceso. Los éxitos obtenidos en U.S. A. en la regulación de hornos, mediante la adaptación de computador de proceso, han de atribuirse a 
lo siguiente:

1) mejor la vigilancia y al mismo tiempo más general la explotación;

2) la aplicación simultánea de un aparato RFA, el cual hace posible la vigilancia de la composición del crudo;

3) un mejor desarrollo del proceso al manejar con detalle todas las magnitudes de influencia.

\section{6. etapas de la automatización}

La automación se verifica, a través de las siguientes etapas ${ }^{5}$ ), que, naturalmente, pueden ser modificadas en casos concretos:

1) equipo con instrumentos de medición;

2) vigilancia desde un puesto central de dirección;

3) automatización de la vigilancia;

4) vigilancia mediante computador.

Las etapas 1) a 3) se han acreditado en toda planificación correcta como medidas de seguridad indicadas para la corrección de perturbaciones.

La vigilancia de la composición del pulverizado en crudo con un aparato RFA y regulador es, evidentemente, muy deseable. Para la regulación automática del horno no hay en realidad necesidad de emplear un computador. Sin embargo, para la dirección del molino es ventajoso el empleo del mismo; y si se aplica con cierto tamaño superior, podrá asumir además la función de un regulador constante para la instalación del horno que pudiera ser necesaria.

El objetivo final es disponer de un gran computador de procesos para la totalidad de la fábrica de cemento. Desgraciadamente, por el momento, los gastos de un computador con su equipo accesorio, que supondría, en total, de 30 a 45 millones de pesetas, hacen problemática su adopción. Sin embargo, en las nuevas construcciones debiera siempre planificarse de manera que se abra la posibilidad de montar en el futuro un computador de procesos, tanto más cuanto no hace falta tenerlo en cuenta al prever el beneficio que se pretende alcanzar en el momento de la inversión.

\section{resumen}

La cantidad de calor que debe suministrarse a un horno rotatorio viene determinada por la composición del crudo, así como por su cantidad. Para una composición y una cantidad de harina cruda constantes, la cantidad de calor suministrada al crudo en todos los puntos del horno debe de ser constante. Estas condiciones pueden ya cumplirse gracias a los reguladores normales de acción continua que aseguran principalmente la constancia cuantitativa de aire y de gases. La regulación del sistema de un horno estará, pues, condicionada por la composición homogénea de la materia cruda, que puede obtenerse por medio del procedimiento de lechos de mezcla o del control de dosificación de componentes en la alimentación del molino. 
El procedimiento de lechos de mezcla es muy caro y no siempre indispensable, empleándose más a menudo el control de dosificación de componentes en la alimentación del molino. Pero en cualquier caso, se hace necesario un procedimiento de análisis rápido. Hoy día, sólo un dispositivo de análisis por rayos $\mathrm{X}$ puede considerarse como práctico para este trabajo. El autor comenta el empleo de los aparatos de análisis por rayos $\mathrm{X}$ de acción continua y discontinua.

Las básculas de bandas dosificadoras aseguran la alimentación constante en crudo, que es importante para la regulación del horno.

En el caso de un horno Lepol, la densidad variable de los gránulos se corrige por una regulación de la velocidad de avance de la parrilla, en función de la altura de los gránulos en la tolva, permaneciendo constante el peso de harina cruda alimentada.

La regulación de la constancia cuantitativa de aire y gases no presenta dificultades cuando las instalaciones están bien estudiadas.

Las condiciones de flujo de aire constante en el enfriador de parrilla se aseguran por regulación directa de la cantidad de aire de enfriamiento o, incluso, manteniendo constantes ciertas presiones o diferencias de presión en el enfriador.

Asegurar la marcha de la fábrica por medio de estaciones centralizadas y la regulación de la composición de la harina cruda gracias al análisis por rayos $\mathrm{X}$ aliada a un computador, representa una meta hacia la que deben tender hoy día todas las fábricas de cemento.

\section{bibliografía}

1 Gringmuth W., Karthaus H., Kinscher H. J.: Praxis der Lepolofenregelung. Zement-Kalk-Gips 9 (1956), 305.

2 LaHL W.: Röntgenfluoreszenzanalyse zur Steuerung und Kontrolle im Zementwerk. Zemen-Kalk-Gips 18 (1965), 78.

3 Henkel F.: Anwendung der RF-Analyse in Schichtlaboratorien. Zement-Kalk-Gips 5 (1965), 253.

4 Bemelman W.: Rohmaterialmischbetten. Zement-Kalk-Gips 19 (1966), 300:

5 BENDY W. R.: Why automate a cement plant? Pit and Quarry 58 (1965), 149 und 155. 